\title{
A Cost-effective Surgical Navigation Solution for Periacetabular Osteotomy (PAO) Surgery
}

\author{
Silvio Pflugi ${ }^{1}$, Li Liu ${ }^{1}$, Timo M. Ecker ${ }^{2}$, Steffen Schumann ${ }^{1}$, Jennifer Cullmann- \\ Bastian $^{3}$, Klaus Siebenrock ${ }^{2}$, Guoyan Zheng ${ }^{1}$ \\ 1 Institute for Surgical Technology and Biomechanics, University of Bern, \\ Switzerland \\ 2 Department of Orthopedic Surgery, Inselspital, University of Bern, Switzerland \\ ${ }^{3}$ Department of Radiology, Inselspital, University of Bern, Switzerland \\ \{silvio.pflugi, li.liu, steffen.schumann, guoyan.zheng\}@istb.unibe.ch, \\ \{TimoMichael.Ecker, Jennifer.CullmannBastian, klaus.siebenrock\}@insel.ch
}

\section{Abstract}

Purpose: To evaluate a low-cost, inertial sensor-based surgical navigation solution for periacetabular osteotomy (PAO) surgery without the line of sight impediment.

Methods: Two commercial inertial measurement units (IMU, Xsens Technologies, The Netherlands), are attached to a patient's pelvis and to the acetabular fragment, respectively. Registration of the patient with a pre-operatively acquired computer model is done by recording the orientation of the patient's anterior pelvic plane (APP) using one IMU. A custom-designed device is used to record the orientation of the APP in the reference coordinate system of the IMU. After registration, the two sensors are mounted to the patient's pelvis and acetabular fragment, respectively. Once the initial position is recorded, the orientation is measured and displayed on a computer screen. A patient-specific computer model generated from a pre-operatively acquired computed tomography (CT) scan is used to visualize the updated orientation of the acetabular fragment.

Results: Experiments with plastic bones ( 8 hip joints) performed in an operating room comparing a previously developed optical navigation system with our inertial-based navigation system showed no statistically significant difference on the measurement of acetabular component reorientation. In all eight hip joints the mean absolute difference was below four degrees.

Conclusion: Using two commercially available inertial measurement units we show that it is possible to accurately measure the orientation (inclination and anteversion) of the acetabular fragment during PAO surgery and therefore to successfully eliminate the line of sight impediment that optical navigation systems have.

Keywords: Computer assisted surgery, inertial measurement unit, navigation system, PAO surgery. 


\section{Introduction}

Chronic abnormal hip mechanics often lead to osteoarthrosis and are associated with instability or impingement stemming from a surgically treatable abnormality (e.g. hip dysplasia). The success of joint-preserving interventions depends on whether the mechanical environment could be normalized and the degree of irreversible articular damage [1]. Hip preservation surgery (e.g. periacetabular osteotomy (PAO)) is performed at early stages of hip diseases when an active lifestyle is demanded. PAO is a demanding surgical procedure for the treatment of adult hip dysplasia [2]. Several cuts separate the acetabular fragment from the rest of the pelvis so that it can be re-oriented to improve femoral coverage. The view of the surgeon during surgery is strongly limited and some cuts have to be made without overseeing the whole area [3].

Recent advancements in computation power, better understanding of the anatomy and new imaging modalities made it possible to merge different innovative technologies like 3D modelling, image registration and instrument tracking to support a physician pre- and intra-operatively in the diagnosis and treatment of pathologies [4]. Surgical navigation for PAO surgery has been done before. Langlotz et al. [5] were one of the first to propose a computed tomography (CT) based PAO navigation system and later, Jäger et al. [6] used a CT based navigation to treat patients with triple osteotomy. Liu et al. [7] proposed a computer-assisted planning and navigation system for PAO surgery including range of motion optimization. Their system offers different modules including a navigation and planning module which allows the virtual simulation of the reorientation procedure including fully automatic detection of the acetabular rim and estimation of the femoral head center. Investigations showed that the use of CAS systems improve accuracy compared to traditional procedures [8-10]. Nevertheless, modern CAS systems are not yet widely used. Surgical navigation is mainly based on optical tracking which has an inherent disadvantage that two cameras need to maintain a line-of-sight to the patient and the instruments, limiting the working area of involved surgeons in the OR [11]. Additionally, the optical stereo camera takes up a lot of space in the already cluttered working area of a surgeon.

Different approaches were presented to overcome these disadvantages. One such approach is electromagnetic tracking $[12,13]$ which is similarly expensive but 
provides lower accuracy than optical tracking due to magnetic field distortions. Recent advancements in microelectromechanical systems (MEMS) made it possible to use inertial sensor-based navigation systems [14-17]. Such inertial measurement units (IMU) are small and comparably cheap and usually consist of a tri-axial gyroscope to measure rotational velocity, a tri-axial accelerometer to measure linear acceleration without gravity and a tri-axial magnetometer to sense the surrounding magnetic field. IMUs use a fusion algorithm that tries to merge information from these sensors in a way to compensate for each sensor's drawback: orientation obtained by the gyroscopes contains very little noise as it gets filtered during the integration step which results in drift. On the other side, accelerometers and magnetometers return very accurate (in the mean) but noisy measurements that can be used to compensate for the gyroscope's drift. This fusion of sensor data is often performed using a variation of the well-known Kalman filter [18].

Behrens et al. [14] proposed an inertial navigation system for bladder endoscopy. They accurately measure angles but experience inaccuracies trying to measure the translation of the endoscope due to temporal drift effects. Ren et al. [17] developed a prototype IMU including an extended Kalman filter for tracking hand-held surgical instruments and compared it to optical tracking. O'Donovan et al. [15] used an inertial and magnetic sensor-based technique for joint angle measurements. The technique makes use of a combination of IMUs attached to the lower extremities to compute joint angles of the ankle joint. All these systems report problems estimating the heading information from the IMU due to magnetic field distortions. Hybrid systems were proposed to overcome the limitations of a single tracking technology [11, 19-21]. Haid et al. [11] presented novel methods combining image processing routines with inertial sensors for surgical navigation and Ren et al. [22] proposed an integrated tracking system for endoscopic surgery combining inertial sensors with electromagnetic tracking to reduce the effect of environmental distortions. However, these systems have an increased complexity and are significantly more expensive.

In this paper, we propose a low-cost system to measure the acetabular fragment during PAO surgery which is solely based on inertial measurement units (IMU). We make use of a newly designed device to measure the orientation of the patient's anterior pelvic plane (APP) and to register the orientation of the patient's 
pelvis with a 3D computer model. Using two IMUs attached to the patient's pelvis and acetabular fragment, respectively, we are able to compute the inclination and anteversion of the acetabular fragment intra-operatively in real-time. Compared to the work reported in [14], we only measure rotations and combine the readings from two IMUs. Walti et al. [23] use a single inertial measurement sensor to track the surgical instrument during pedicle screw placement. Their application makes use of one sensor and the whole procedure to place a pedicle screw only takes a short amount of time reducing the influence of drift errors. IMUs usually have built-in mechanisms to compensate for magnetic field distortions. One possibility is to rely more on the gyroscope readings during time of disturbance which results in a drift of the heading value. We decided to implement our own Kalman filter as the built-in filtering algorithm provided by the sensor company relies heavily on the drift-prone gyroscope data in times when the magnetic field environment differs significantly from what is expected outdoors [24]. Such mechanisms to compensate for disturbed magnetic field environments are useful if disturbances are expected or if the magnetic field changes in space. Our work is based on the assumption that the magnetic field in the OR is different than outdoors but does not change in time and is stable within the small operating volume of our sensors. This allows us to generally rely more on the magnetometer values which are noisy but more accurate in the mean than to rely on gyroscope readings which would be less noisy but, due to the integration step, experience drift.

Our work can be compared to [15] with the difference that we use the system in a clinical setting inside the OR for a demanding surgery. We also investigated our assumption of a stable magnetic field environment in the OR which often influences the performance of IMUs especially when they are used over a prolonged period as is the case for PAO surgery. To our knowledge, we are the first to use IMUs to measure the acetabular orientation during PAO surgery, successfully removing the line-of-sight limitation of optical tracking systems.

\section{Materials and Methods}

\section{Pre-operative Steps}

For visualization purposes a patient-specific computer model which is acquired from segmented CT data (using AMIRA, Visage Imaging, San Diego, USA) is 
used (see

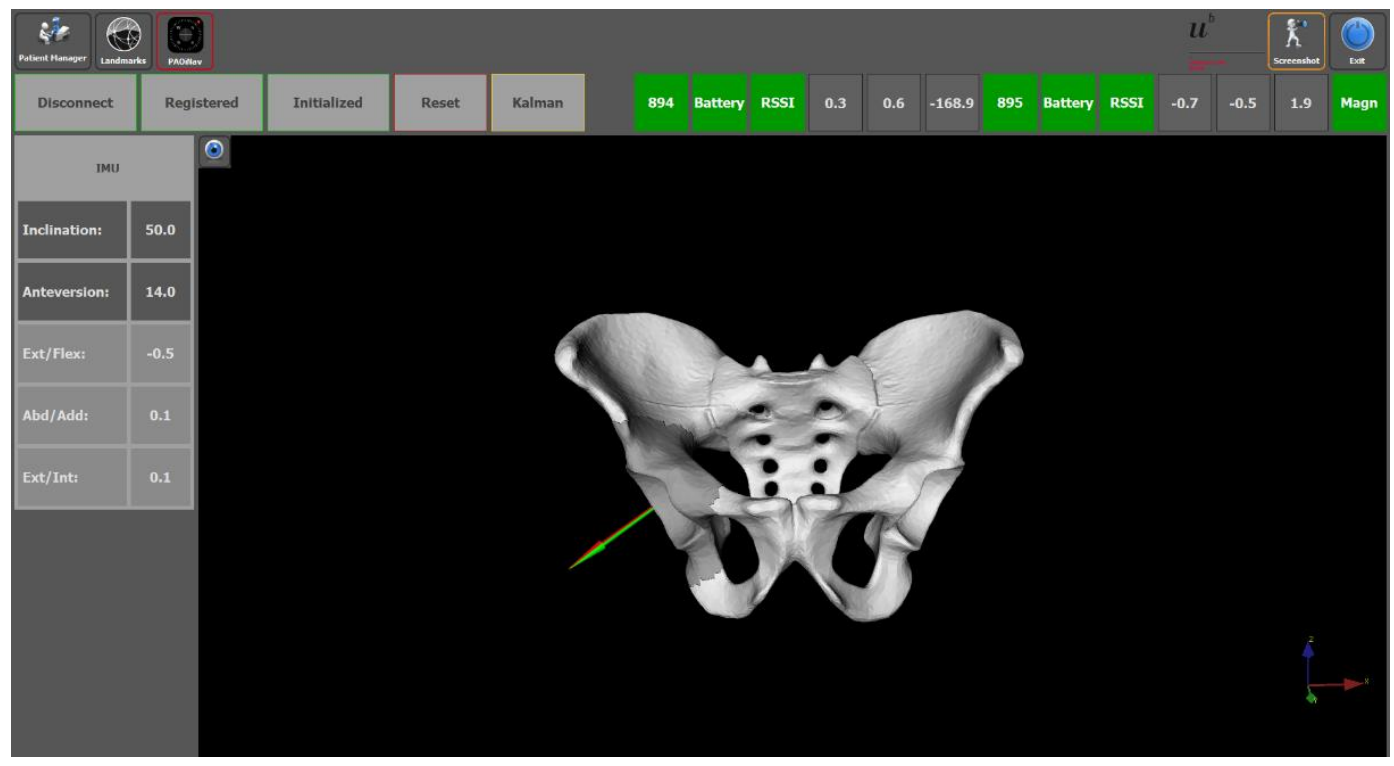

Figure 9). Next, four landmarks are picked on the computer model before the surgery. These landmarks - left and right anterior superior iliac spine (ASIS) as well as the left and right pubic tubercles (PUBIS) - define the anterior pelvic plane (APP) which is used later for registration. Re-orientation of the acetabular fragment is modeled as a rotation of the fragment around the center of the femoral head. With the help of a previously developed comprehensive PAO planning system [7] we estimate the femoral head center by fitting a sphere to the femoral head. Additionally, to compute acetabular orientation (inclination and anteversion) the acetabular cup plane normal has to be known (Figure 5). To compute the cup plane, the PAO planning system fully automatically detects the acetabular rim points and a plane is fitted through the points to get the plane normal [7].

\section{Inertial Measurement Units}

Two commercially available wireless MEMS inertial measurement units (Xsens Technologies, The Netherlands) fuse data from three internal sensors, namely three accelerometers providing linear acceleration (without gravity), three gyroscopes providing rotational velocity and three magnetometers providing the magnetic field as well as a barometer to output full 3D orientation data in an Earth-fixed coordinate system. The sensor fusion is performed using a variation of a Kalman filter [18] as described below. 


\section{Kalman Filter}

A linear Kalman filter was implemented to compute the orientation of each sensor. The input to the filter was the calibrated data from the sensors provided by the company's software development kit (SDK). "Calibrated" in this case means that the data is compensated for misalignment of the internal sensor units (tri-axial sensors) and temperature. Our application is implemented in $\mathrm{C}++$ and follows the well-known Kalman filtering approach with a state vector $\overrightarrow{\mathrm{x}}$ and a measurement and process model which relates the state at a previous time with the current state [18]:

$$
\begin{aligned}
& \overrightarrow{\mathrm{x}}=\left(x, y, z, G_{x}, G_{y}, G_{z}, A_{x}, A_{y}, A_{z}\right)^{T} \\
& \vec{x}_{k}=F \vec{x}_{k-1}+\vec{w}_{k-1}
\end{aligned}
$$

The state vector $\vec{x}$ contains the positional data $(x, y, z)$ with gyroscope data $\left(G_{x}, G_{y}, G_{z}\right)$ and accelerometer data $\left(A_{x}, A_{y}, A_{z}\right)$ from the sensor (Equation 1). The state transition matrix $F$ and the process noise $\vec{w}$ with covariance $Q$ are used to compute the current state $\vec{x}_{k}$ at time point $k$ (Equation 2). The measurement model is:

$$
\vec{z}_{k}=H \vec{x}_{k}+\vec{v}_{k}
$$

The matrix $H$ relates the current state with the measurements $\vec{z}_{k}$, and $\vec{v}$ is the normal distributed measurement noise with covariance $R . Q$ and $R$ are diagonal matrices and were empirically determined to have diagonal elements of 0.1 for $Q$ and 0.001 for $R$. Based on this model, we use the general Kalman filter equations consisting of a prediction (Equation 4 and 5) and correction step (Equation 6,7 and 8 , see Figure 4) to compute the Kalman gain $K_{k}$, which weighs the measurement against the prediction, and to update the state estimate $\vec{x}_{k}$ and covariance $P_{k}[24]$ :

$$
\begin{aligned}
& \vec{x}_{k}^{-}=F \vec{x}_{k-1} \\
& P_{k}^{-}=F P_{k-1} F^{T}+Q \\
& K_{k}=P_{k}^{-} H^{T}\left(H P_{k}^{-} H^{T}+R\right)^{-1} \\
& \vec{x}_{k}=\vec{x}_{k}^{-}+K_{k}\left(\vec{z}_{k}-H \vec{x}_{k}^{-}\right) \\
& P_{k}=\left(I-K_{k} H\right) P_{k}^{-}
\end{aligned}
$$




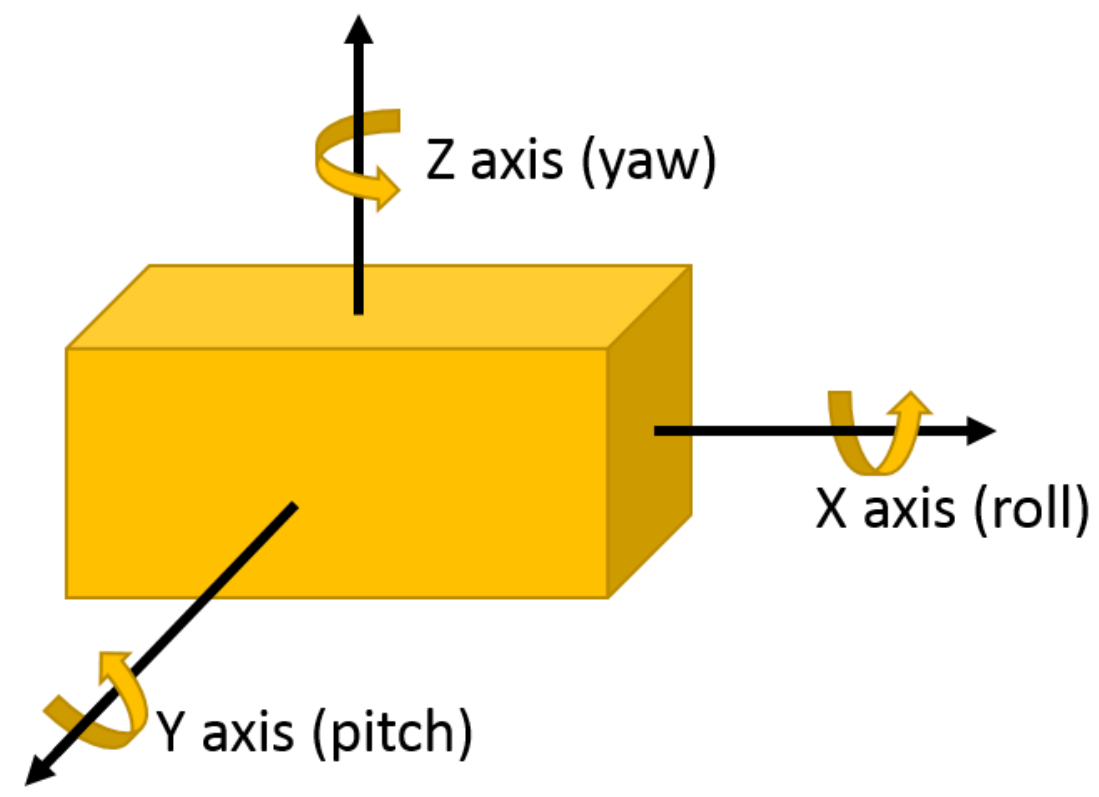

Figure 3) based on the current state vector (Equation 3). Roll and pitch can be computed using the values from the accelerometer:

$$
\begin{aligned}
& r=\arctan \left(A_{y}, A_{x}\right) \\
& p=\arctan \left(-A_{x}, \sqrt{A_{y}^{2}+A_{z}^{2}}\right)
\end{aligned}
$$

For the heading value we must take the tilt of the sensor into account and compensate for it. This can be done using the previously computed roll and pitch values:

$$
\begin{gathered}
x h=M_{z} * \sin (r)-M_{y} * \cos (r) \\
y h=M_{x} * \cos (p)+M_{y} * \sin (r) * \sin (p)+M_{z} * \sin (p) * \cos (r) \\
y a=\arctan (x h, y h)
\end{gathered}
$$

In Equation 11, 12 and 13, $M_{x}, M_{y}, M_{z}$ are the calibrated raw values from the magnetometer. The three values computed from the accelerometer and magnetometer readings for roll, pitch and yaw represent the measurements $\vec{z}_{k}$ in Equation 3. An overview of the Kalman filter can be seen in Figure 4.

\section{Anatomy Registration}

After acquiring all pre-operative information, the computer model's orientation is registered to the patient's pelvis' orientation. For that task, we designed a new device ( 


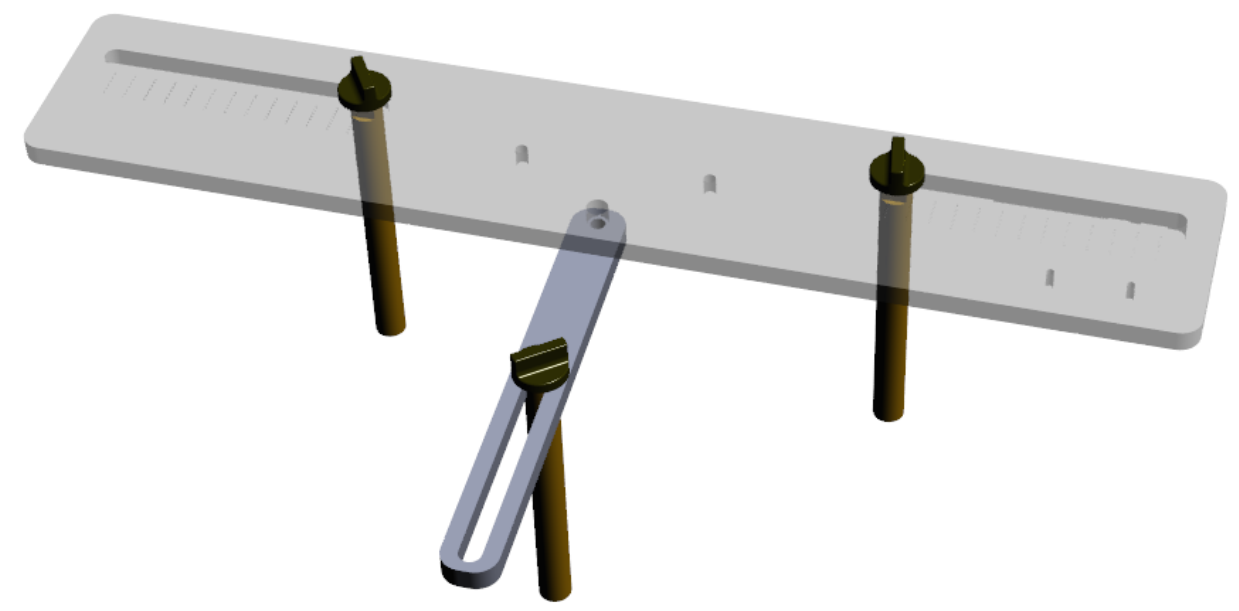

Figure 1 and Figure 2) which aligns a single IMU with the patient's APP. Aligned with the device's top plate, the sensor's orientation (local z-axis) represents the APP normal. The computer model is then transformed in a way that its APP (known from the picked landmarks), and therefore the whole model, has the same orientation as the patient's APP/pelvis. One hundred data packets from the wireless sensor (quaternion orientations) are recorded and the orientation of the APP is estimated by taking the mean of all quaternion orientations to filter out noise from the data. The algorithm proposed by Markley et al. [25] is used to compute the average quaternion by performing an eigenvalue/eigenvector decomposition of a matrix composed of the given quaternions.

\section{Sensor setup}

After registration, the two sensors are mounted to the patient: one sensor is attached to the patient's pelvis (Sensor A) and the other is mounted to the acetabular fragment (Sensor B, see Figure 6). The output from sensor $A$ and $B$ at time $t$, each represented using a rotation matrix, specifying the orientation of a sensor with respect to an Earth-fixed reference orientation $O$ is then:

$$
\begin{aligned}
& R_{O A_{t}}=R_{A_{\text {yaw }}} * R_{A_{\text {pitch }}} * R_{A_{\text {roll }}} \\
& R_{O B_{t}}=R_{B_{\text {yaw }}} * R_{B_{\text {pitch }}} * R_{B_{\text {roll }}}
\end{aligned}
$$

With $R_{O A_{t}}$ and $R_{O B_{t}}$ being the rotation matrices of sensor $A$ (pelvis) and $B$ (fragment) at time $t$ representing the rotations necessary to bring sensor $A$ and $B$ from the reference orientation $O$ (perfectly aligned with the Earth-fixed coordinate 
system) to its current orientation. The rotation is expressed as rotations around the three axes of the Earth fixed coordinate system (roll $-\mathrm{x}$-axis, pitch $-\mathrm{y}$-axis and yaw - z-axis, see

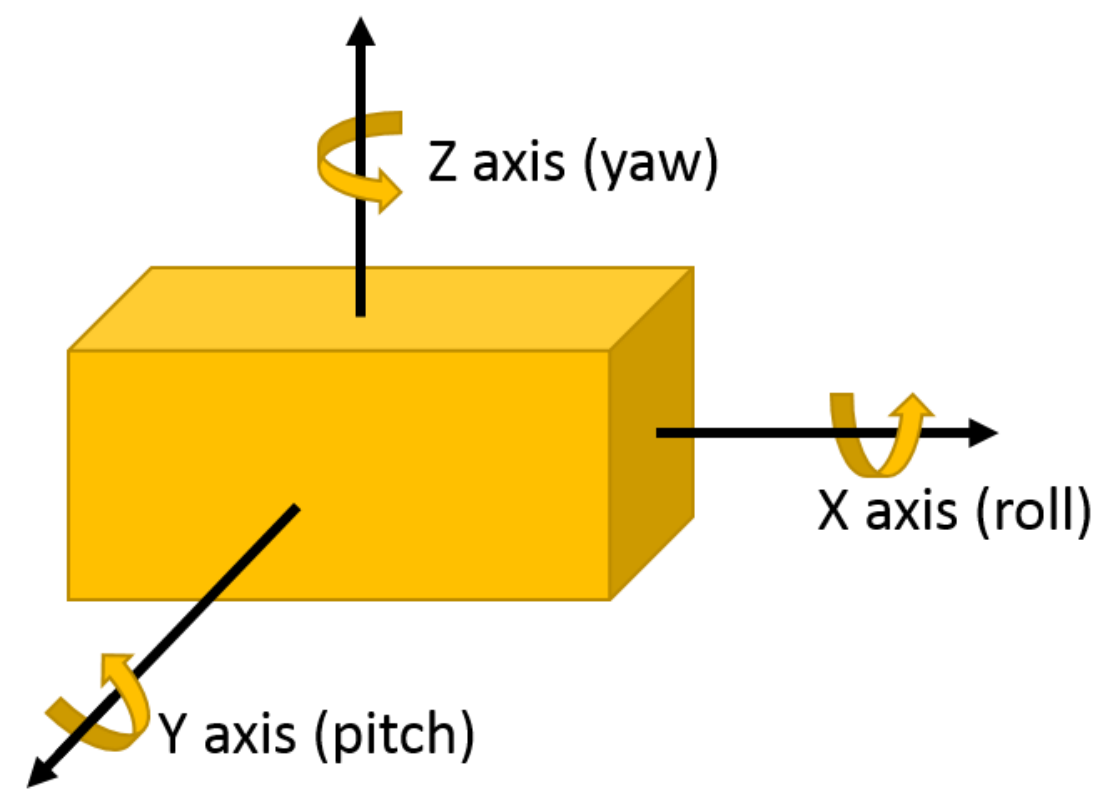

Figure 3). Before reorientation, the starting orientation of each sensor is recorded $(t=0)$. At every time point $t$ after the initialization step $(t=0)$, we take the current rotation matrices from sensor $A$ and $B$ to compute the incremental update between $t-1$ and $t$ for each sensor:

$$
\begin{aligned}
& R_{d t_{A}}=R_{O A_{t}} * R_{O A_{t-1}}^{T} \\
& R_{d t_{B}}=R_{O B_{t}} * R_{O B_{t-1}}^{T}
\end{aligned}
$$

Intuitively speaking, $R_{d t_{A}}$ and $R_{d t_{B}}$ defines a rotation from the sensor's orientation at time point $t-1$ back to the reference orientation $O$ and then forward to the orientation at time $t$. To estimate the rotation of the fragment with respect to the patient's pelvis we must account for movements of the pelvis during surgery. Under the assumption that the rotation center for pelvis and fragment is the same, the rotation of the pelvis (Sensor $A$ ) is the same as an inverse rotation of the fragment (Sensor B) and vice versa. This way, movements of the patient intraoperatively can be compensated by treating the incremental update of the pelvis $R_{d t_{A}}$ inversely. Therefore, to compute the incremental update of the fragment, we first rotate the fragment by the incremental update from sensor $B$ and then apply an inverse rotation using the incremental update from sensor $A$ :

$$
R_{F_{d t}}=R_{d t_{A}}^{T} * R_{d t_{B}}
$$


Using $R_{F_{d t}}$, we rotate the acetabular cup plane normal at every time point $t$ and recompute the acetabular orientation as described below.

\section{Acetabular Orientation}

In our application we compute radiographic inclination and anteversion angles [26] (Figure 5), which are defined as:

$$
\begin{aligned}
& \text { inclination }=r 2 d\left(\operatorname{acos}\left(\operatorname{dot}\left(\overrightarrow{\operatorname{proj}},-\vec{n}_{\text {axial }}\right)\right)\right) \\
& \text { anteversion }=r 2 d\left(\operatorname{acos}\left(\operatorname{dot}\left(\overrightarrow{\operatorname{proj}}, \vec{n}_{\text {cup }}\right)\right)\right)
\end{aligned}
$$

Where $r 2 d$ is the function to convert from radians to degree, $\vec{n}_{c u p}$ is the normal of the acetabular cup plane defined by the rim points, $\vec{n}_{\text {axial }}$ represents the axial plane which is defined as the cross product between the APP normal and the vector connecting the right and left anterior superior iliac spine and $\overrightarrow{p r o j}$ is defined as follows:

$$
\overrightarrow{\operatorname{proj}}=\vec{n}_{c u p}-\operatorname{dot}\left(\vec{n}_{c u p}, \vec{n}_{A P P}\right) * \vec{n}_{A P P}
$$

Using $R_{F_{d t}}$ (Equation 16) we are able to update the orientation of the acetabular cup plane at every time point and then re-compute the necessary plane normals and vectors to compute the inclination and anteversion values (Figure 5). The overall procedure is outlined below.

- Pre-operative Steps

- Acquire CT and reconstruct a patient specific 3D computer model of the hip and proximal femur

- Extract the acetabular rim points from the computer model and fit a plane through these points to get the acetabular cup plane normal [7]

- Fit a sphere to the femoral head and extract the center of this sphere as the rotation center

- Pick landmarks on the 3D computer model

- Intra-operative Steps

- Connect to both sensors

$\circ$ Measure the orientation of the APP using the new measurement device

- Mount the two sensors to the patient's pelvis and the acetabular fragment and record initial orientation relative to each other

o Start re-orientation 


\section{Experiments}

In order to validate our system, we performed a plastic bone study using 8 hip joints (4R, 4L, four different pelvis models). The experiments were performed inside an operating room (OR) to simulate an as-real-as-possible magnetic-field environment for the sensors. For each pelvis, a CT scan was acquired and the 3D computer model was segmented. The osteotomies were directly drawn onto the plastic bones by an experienced orthopaedic surgeon (TME) and then cut using a coping saw. For comparison and validation purposes, we simultaneously ran a previously developed navigation system using an optical tracking camera (Polaris, NDI Canada) as ground truth [7]. The optical tracking system works the same way as the sensor application, only considering rotations and not translation. APP registration is performed by picking and digitizing the same landmarks (right and left ASIS and both pubic tubercles). After registering the pelvis' APP with the model and recording the starting orientation, the acetabular fragment was slowly rotated to new positions and every seven seconds the inclination and anteversion values as well as the current fragment rotation matrix were recorded from both systems simultaneously. The recorded rotation matrix allows us to better compare the two systems as anteversion and inclination represent a projected 2D angle and the rotation matrix gives us a better estimate of the accuracy of our Kalman filter. Instead of comparing the rotation matrices directly, we converted the rotation matrices to unit quaternions. $q_{\text {ins }}$ represents the acetabular orientation measured by the IMUs and $q_{\text {ots }}$ is the unit quaternions measured by the optical tracking system. Equation 20 shows the relationship between these two unit quaternions at any time point:

$$
0 \leq \operatorname{abs}\left(\operatorname{dot}\left(q_{\text {ins }}, q_{o t s}\right)\right) \leq 1
$$

If two unit quaternions represent the same orientation, then Equation 20 is equal to 1 . We use this relationship as a metric to compare the two systems. We evaluated Equation 20 for all measurements and report the mean and standard deviation. Additionally, to investigate our assumption of a stable magnetic field environment, we also automatically recorded the raw magnetic field data every 20s from each sensor during the whole procedure. Applying the inverse orientation matrix of the sensor to the magnetic field vector measured by the same 
sensor will transform it to Earth coordinate system. If our assumption holds, this should be constant throughout the procedure.

\section{Statistical Evaluation}

To evaluate the feasibility of our inertial sensor-based PAO navigation system for measuring acetabular orientation we defined the following hypotheses:

1. The measurements performed during surgery (anteversion, inclination) of our inertial sensor-based application are not significantly different than the measurements performed using the optical tracking-based system during the same procedure.

2. The mean absolute difference between our system and the optical trackingbased system is less than five degrees.

For the first null hypothesis we treat anteversion and inclination values separately and compare them to the optical tracking-based system using a Wilcoxon rank sum test and correlation between the two vectors. The first null hypothesis will be rejected if the p-value is smaller than 0.05 or the correlation is lower than 0.9 . The second null hypothesis will be rejected if the mean absolute difference between the two systems is higher than five degrees for anteversion or inclination. Five degrees as a threshold was chosen based on input from our clinical partners to be a reasonable cutoff to evaluate accuracy. Statistical evaluation was performed using Matlab (Mathworks, Natick, MA, USA).

\section{Results}


Table 1 and Table 2 show the results for all eight hip joints (inclination and anteversion). As for the first null hypothesis, the results from both systems are for all eight hip joints not statistical significantly different (p-value > 0.05). Consistently, the correlation is larger than 0.9 in all eight cases and therefore we can accept the first null hypothesis for all hip joints. We can also accept the second null hypothesis as the mean absolute difference was lower than the beforehand defined five degrees in all eight hips for both inclination and anteversion. An example case showing the inclination and anteversion over time for one hip joint (2L) is shown in Figure 7 and Figure 8. 
Table 3 shows the comparison of the rotation matrices using the quaternion representation and the standard deviations of the measured magnetic field vector for both sensors (attached to pelvis and to the fragment). In all eight hip joints, the acetabular orientation measured by the two systems is strongly correlated. The evaluation of the magnetic field environment shows that our assumption of a stable magnetic field is valid. There are no big fluctuations noticeable for both sensors and all hips during reorientation.

\section{Discussion}

In this work, we demonstrated the feasibility of a low-cost system using IMUs to measure the orientation of the acetabular fragment during PAO surgery, which we compared to the current gold-standard, optical tracking. We defined two hypotheses which were accepted in all cases. Our Kalman filter does not incorporate any mechanism to compensate for magnetic field disturbances. If a ferromagnetic material would come too close to one sensor, the values would not be reliable. However, for PAO surgery, the surgeon cuts the fragment beforehand and there is usually no need for any instruments to interfere with the reorientation process. And even if there is the need to use a ferromagnetic object, the correct values would bounce back after the object is removed. The assumption of a stable magnetic field environment is valid in the OR especially for the limited rotation range necessary to measure the reorientation. The magnetic field vector for each sensor in Earth-coordinate system was stable throughout the reorientation process. As can be seen in Figure 7 and Figure 8, the maximum difference between the two systems is sometimes quite high, increasing the mean absolute difference. The large differences may be attributed to the fact that for the person holding the acetabular fragment it is sometimes difficult to keep a steady position for several seconds. However, this may not have an influence in a real surgery: the acetabular fragment can be better kept in a certain orientation for a couple of seconds to check the current orientation parameters since it is stabilized by the femoral head and the joint capsule even though it is completely separated from the pelvis.

The main concern on using IMUs is their systematic error due to temporal drift effects that occur from integrating raw measurements over time [14]. O'Donovan et al. [15] report differences in accuracy depending on if the measured joint angle is mainly based on the sensor's heading information or not and therefore relying 
on a stable magnetometer measurement. Ren et al. [17] also report that the magnetometer is vulnerable to environmental interferences. The first couple of seconds, the sensors estimate the quality of the magnetic environment and the Kalman filter adjusts to small magnetic field distortions by trusting less on the magnetometer output and relying more on the output of the gyroscope to compute heading information. This occurs even if the magnetic field is stable but different than expected at the current location (based on computed angle between magnetic field and Earth surface [24]). The sensor needs some time to adjust internally and output a stable heading value. According to the manufacturer, the necessary time for the Kalman filter to warm-up is less than two minutes. In our experience, this is not enough to get stable measurements for the IMUs heading value using the company's internal filtering algorithm. We set the warm-up time to 12 minutes which resulted in a stable yaw angle even in an OR. This over-compensation would not be necessary in our case, as the range of motion is very limited and no ferromagnetic objects are expected to interfere during reorientation. Therefore we decided to implement our own version of a Kalman filter which does not include such a mechanism.

We register the patient's APP with the computer model using a newly designed device (

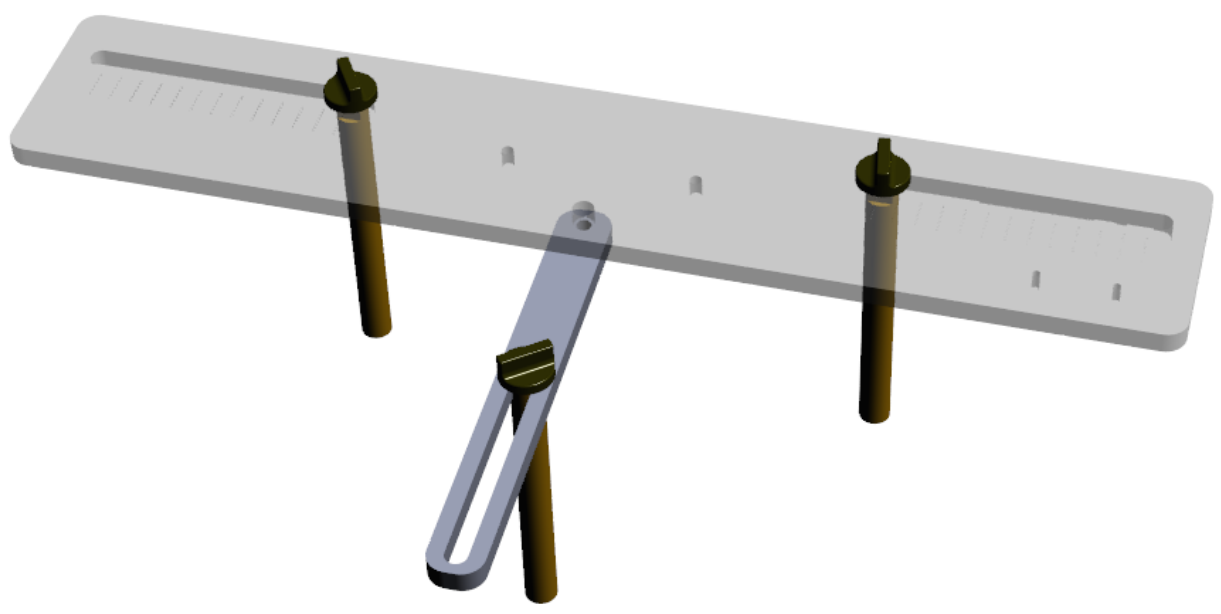

Figure 1). It has three levers which are placed on the patient's left and right ASIS and one pubic tubercle (Figure 2). This registration method is very convenient but might not always be that accurate in a real surgery due to an unknown amount of soft tissue between the bone and the skin surface. The unknown amount of soft tissue is not an error specific to our application as it is a general source of error 
also for optical navigation-based system that use palpation to digitize landmarks [27]. In our plastic bone study, this problem is not apparent since we can directly place the device onto the necessary bony structures.

Our system successfully removes the line of sight impediment of optical trackingbased navigation systems. While our hypotheses could be accepted for all experiments, the accuracy that can be achieved using IMUs is measurably lower than what camera-based systems offer. Nevertheless, the increased convenience and low-cost of our proposed system may help to further push navigation systems into clinical routine.

\section{Conclusion}

In this work we proposed a low-cost system to measure the orientation of the acetabular fragment during PAO surgery. We showed the feasibility of using IMUs in a surgical environment using a plastic bone study performed in an OR. Future work will include a cadaver study to validate the clinical usefulness of the proposed system and further investigation of ways how to handle magnetic field disturbances.

\section{Conflict of Interest}

The authors declare that they have no conflict of interest.

\section{Informed consent}

Informed consent was obtained from all individual participants included in the study.

\section{References}

1. Millis M, Kim Y. Rationale of Osteotomy and Related Procedures for Hip Preservation: A Review. Clinical Orthopaedics \& Related Research. 2002;405:108-21.

2. Hsieh $\mathrm{PH}$, Chang $\mathrm{YH}$, Shih $\mathrm{CH}$. Image-guided periacetabular osteotomy: computer-assisted navigation compared with the conventional technique: a randomized study of 36 patients followed for 2 years. Acta orthopaedica. 2006;77(4):591-7. doi: 10.1080/17453670610012656. PubMed PMID: 16929435. 3. Langlotz F, Stucki M, Baechler R, Scheer C, Ganz R, Berlemann U, Nolte L-P, Mueller M. The First Twelve Cases of Computer Assisted Periacetabular Osteotomy. Computer Aided Surgery. 1997;2:317-26. 
4. Khanduja V, Villar R. Arthroscopic Surgery of The Hip - Current Concepts and Recent Advances. J Bone Joint Surg Br. 2006;88-B(12):1557-66. doi: 10.1302/0301-620x.88b12.

5. Langlotz F, Bachler R, Berlemann U, Nolte LP, Ganz R. Computer Assistance for Pelvic Osteotomies. Clin Orthop. 1998;354:92-102.

6. Jäger M, Westhoff B, Wild A, Krauspe R. Computer-assisted Periacetabular Triple Osteotomy for Treatment of Dysplasia of the Hip. Zeitschrift fur Orthopadie und Ihre Grenzgebiete. 2004;142(1):51-9. 7. Liu L, Ecker T, Schumann S, Siebenrock KA, Nolte LP, Zheng G. Computer Assisted Planning and Navigation of Periacetabular Osteotomy with Range of Motion Optimization. MICCAI. 2014;2:643-50.

8. Jolles D, Genoud P, Hoffmeyer P. Computer assisted cup placement techniques in total hip arthroplasty improve accuracy of placement. Clin Orthop. 2004;426(1):174-9.

9. Nogler M, Kessler O, Prassl A. Reduced variability of acetabular cup positioning with use of an imageless navigation system. Clin Orthop. 2004;426(1):159-63.

10. Ryan JA, Jamali AA, Bargar WL. Accuracy of computer navigation for acetabular component placement in THA. Clinical orthopaedics and related research. 2010;468(1):169-77. doi: 10.1007/s11999-009-1003-7. PubMed PMID: 19629609; PubMed Central PMCID: PMC2795805.

11. Haid M, Kamil M, Chobtrong T, Guenes E. Machine-Vision-Based and Inertial-Sensor-Supported Navigation System for the Minimal Invasive Surgery. AMA Conferences 2013 - SENSOR 2013. 2013. doi: 10.5162/sensor2013/P5.3. 12. von Jako R, Carrino J, Yonemura K, Noda G, Zhue W, Blaskiewicz D, Rajue M, Groszmann D, Weber G. Electromagnetic navigation for percutaneous guide-wire insertion: Accuracy and efficiency compared to conventional guidance. NeuroImage. 2009;47(2):127-32.

13. Zhang H, Banovac F, Lin R, Glossop N, Wood B, Lindisch D, Levy E, Cleary K. Electromagnetic tracking for abdominal interventions in computer aided surgery. Computer Aided Surgery. 2006;11(3):127-36.

14. Behrens A, Grimm J, Gross S, Aach T. Inertial Navigation System for Bladder Endoscopy. Engineering in Medicine and Biology Society, EMBC 2011. 2011.

15. O'Donovan KJ, Kamnik R, O'Keeffe DT, Lyons GM. An inertial and magnetic sensor based technique for joint angle measurement. Journal of biomechanics. 2007;40(12):2604-11. doi: 10.1016/j.jbiomech.2006.12.010. PubMed PMID: 17346716.

16. Rebello K. Application of MEMS in Surgery. Proc IEEE. 2004;92(1):43-

55.

17. Ren H, Kazanzides P. Investigation of attitude tracking using an integrated inertial and magnetic navigation system for hand-held surgical instruments. IEEE/ASME TRANSACTIONS ON MECHATRONICS. 2012;17(2):210-7.

18. Kalman R. A New Approach to Linear Filtering and Prediction Problems. Transaction of the ASME - Journal of Basic Engineering. 1960:35-45.

19. Beller S, Eulenstein S, Lange T, Hunerbein M, Schlag PM. Upgrade of an optical navigation system with a permanent electromagnetic position control: a first step towards "navigated control" for liver surgery. Journal of hepato-biliarypancreatic surgery. 2009;16(2):165-70. doi: 10.1007/s00534-008-0040-z. PubMed PMID: 19183828. 
20. Claasen G, Martin P, Picard F. High-Bandwidth Low-Latency Tracking Using Optical and Inertial Sensors. Proceedings of the 5th International Conference on Automation, Robotics and Applications. 2011:366-71.

21. Mahfouz M, Kuhn M, To G, Fathy A. Integration of UWB and Wireless Pressure Mapping in Surgical Navigation. IEEE TRANSACTIONS ON MICROWAVE THEORY AND TECHNIQUES. 2009;57(10):2550-64.

22. Ren H, Rank D, Merdes M, Stallkamp J, Kazanzides P. Multisensor Data Fusion in an Integrated Tracking System for Endoscopic Surgery. IEEE Transactions On Information Technology in Biomedicine. 2012;16(1):106-11. 23. Walti J, Jost G, Cattin P. A New Cost-Effective Approach to Pedicular Screw Placement. MICCAI - Lecture Notes in Computer Science. 2014(8678):907.

24. Roetenberg D, Luinge H, Veltlink P. Inertial and magnetic sensing of human movement near ferromagnetic materials. Proceedings of the 2nd IEEE/ACM International Symposium on Mixed and Augmented Reality. 2003. 25. Markely F, Cheng Y, Crassidis J, Oshman Y. Averaging Quaternions. Journal of Guidance, Control, and Dynamics. 2007;30(4):1193-6.

26. Murray D. The definition and measurement of acetabular orientation. J Bone Joint Surg Br. 1993;75-B(2):228-32.

27. Richolt J, Effenberger H, Rittmeister M. How does soft tissue distribution affect anteversion accuracy of the palpation procedure in image-free acetabular cup navigation? An ultrasonographic assessment. Computer Aided Surgery. 2005;10(2):87-92. 


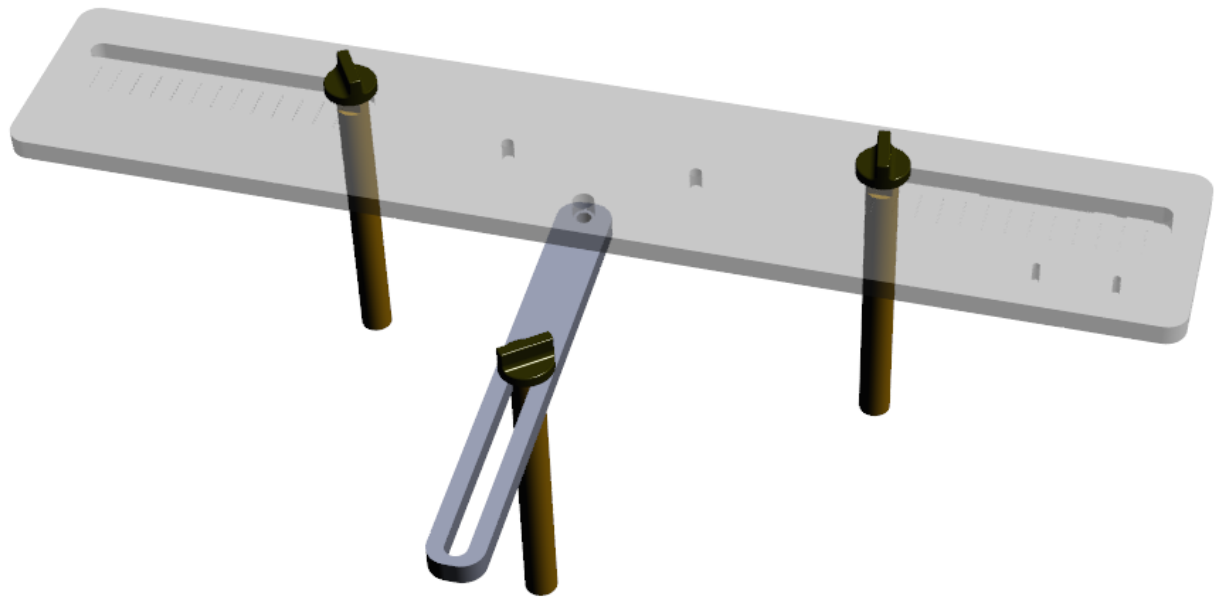

Figure 1: APP measuring device. The three pillars are placed on the right and left anterior superior iliac spine (ASIS) and one of the two pubic tubercles. The sensor is placed on the top plate which is aligned with the APP.

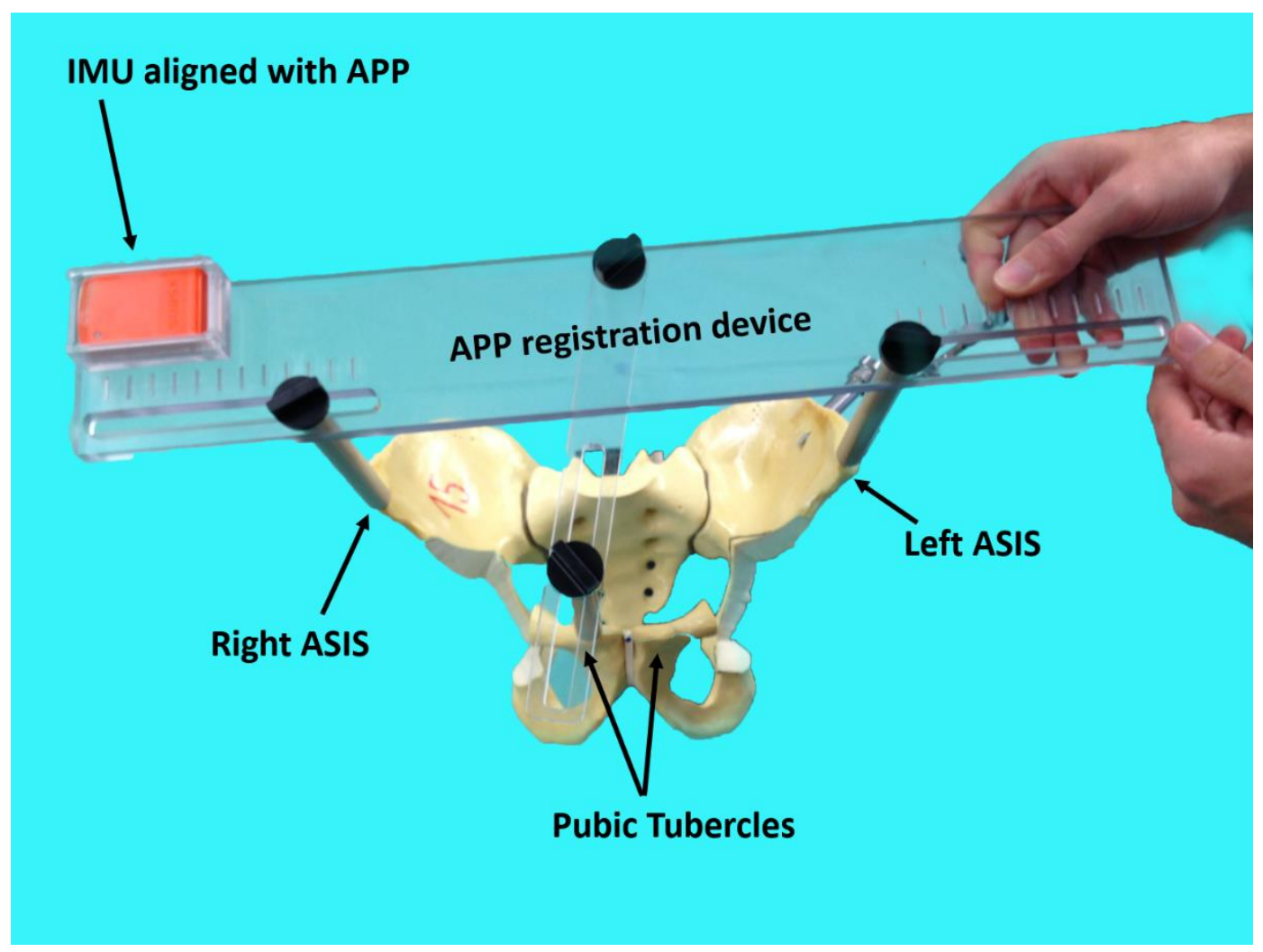

Figure 2: The newly designed APP measuring device is placed on the patient's pelvis so that the three levers are placed on the left and right anterior superior iliac spines as well as on one of the two pubic tubercles. The top plate will then be aligned with the APP and the sensor can measure the orientation of it. 


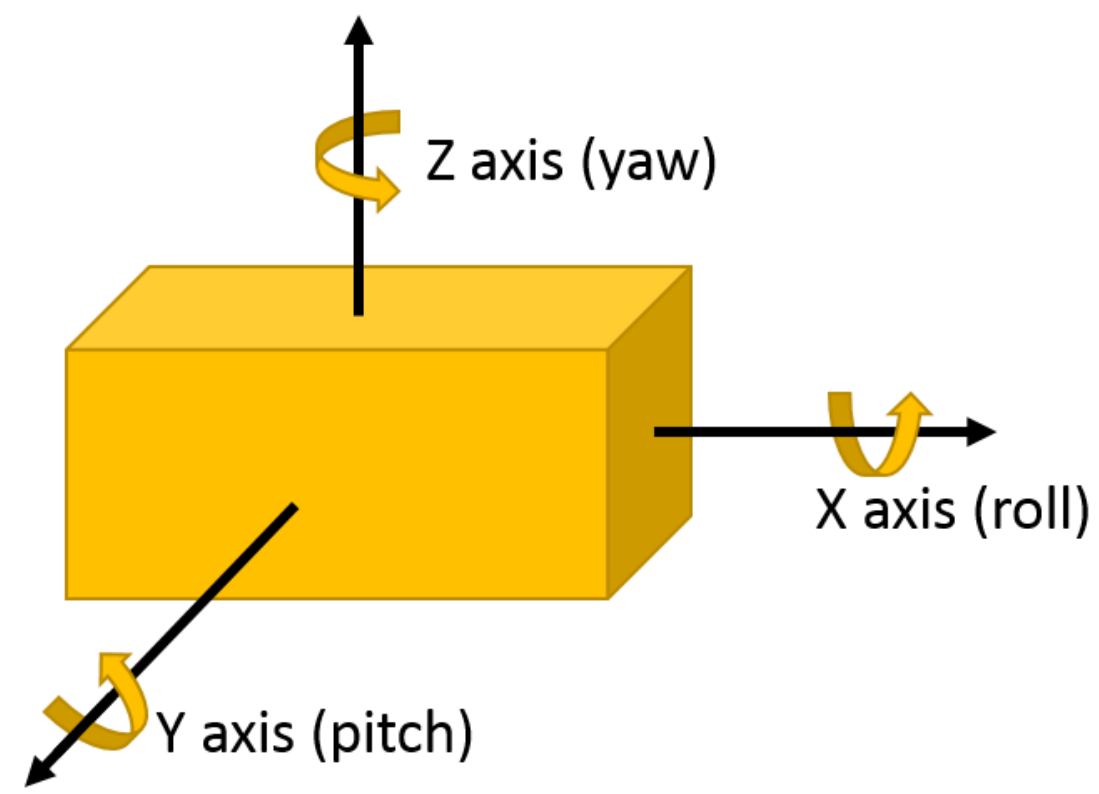

Figure 3: The three angles around the main axes are roll (rotation around x-axis), pitch (rotation around $y$-axis) and the heading value or yaw angle (rotation around z-axis).

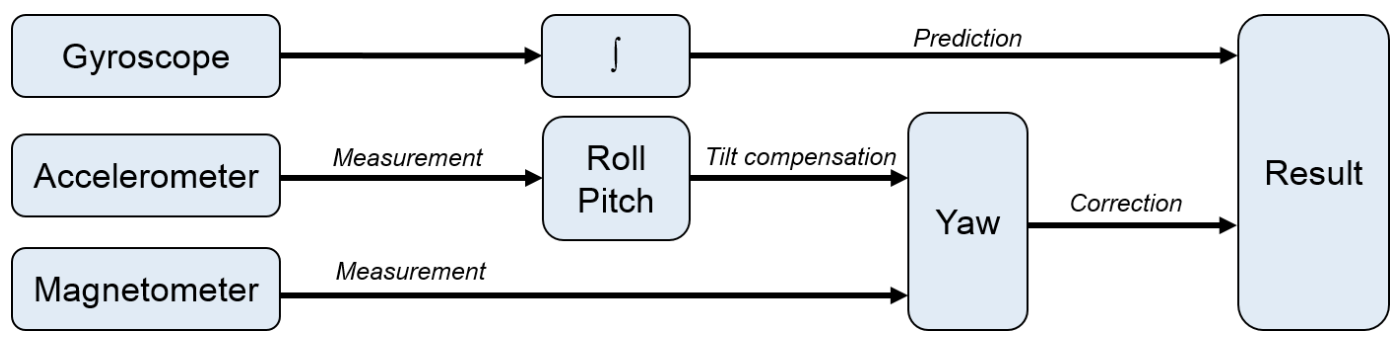

Figure 4: An overview of our Kalman filter implementation. The gyroscope signal is integrated to predict the new orientation. The accelerometers readings are used to compute roll and pitch which are then used for tilt compensation when computing the yaw value using magnetometer readings. These measurements are then used to correct the predicted orientation from the gyroscope readings to return the final orientation. 


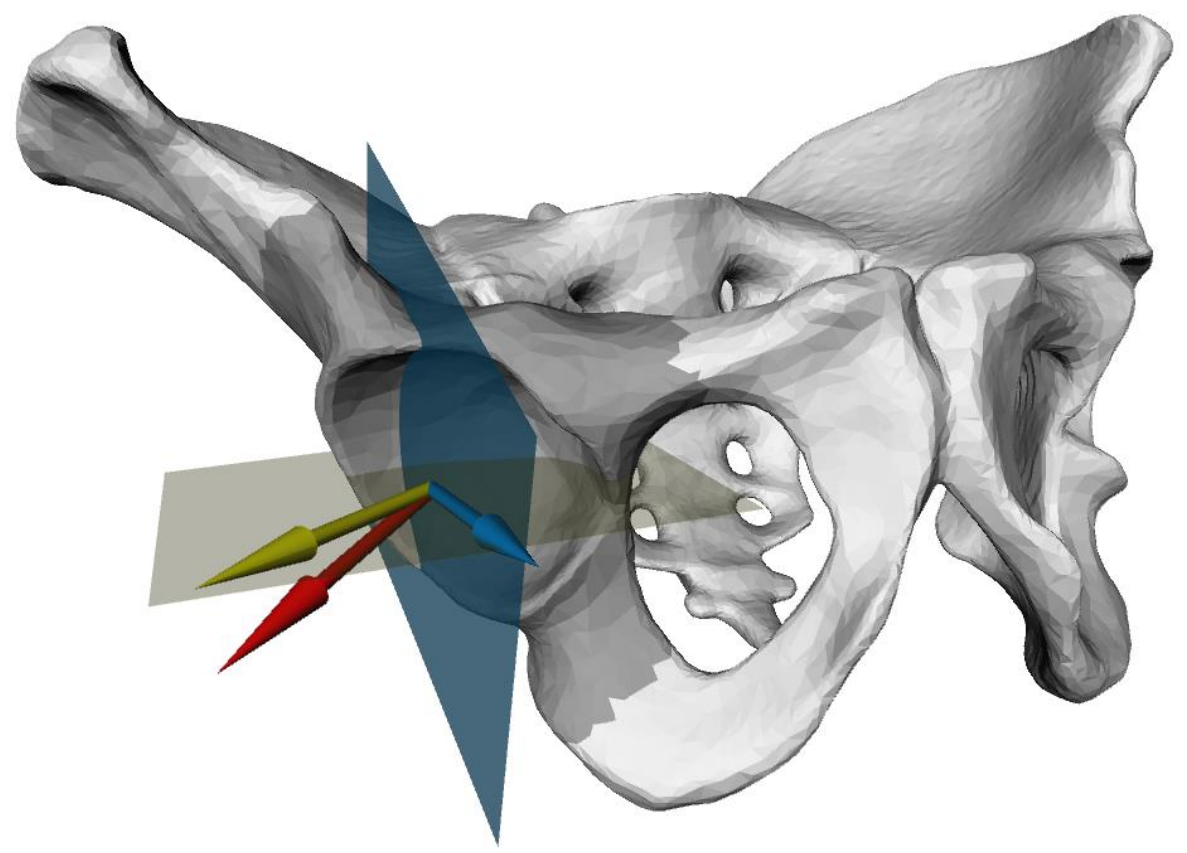

Figure 5: Visualization of inclination and anteversion angles. The red arrow represents the acetabular cup normal, yellow represents the projection of the cup normal onto the anterior pelvic plane (APP, yellow plane). Blue is the projection of the yellow vector on to the sagittal plane (blue plane). Inclination is defined as the angle between the yellow and blue vector and anteversion is defined as the angle between the red and yellow vector.

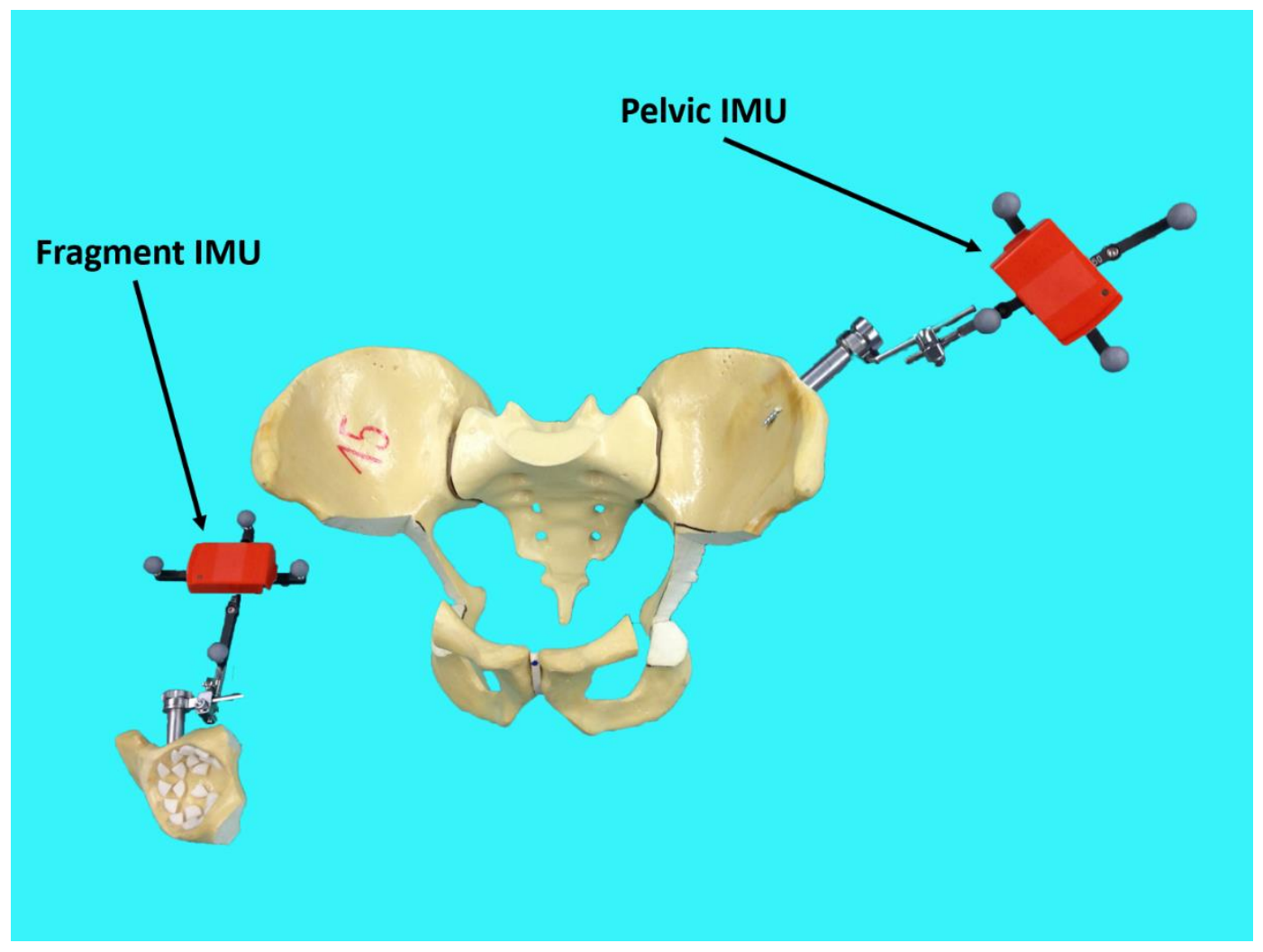


Figure 6: The sensor setup with one IMU attached to the acetabular fragment and the other to the pelvis. In our setup, we attached the IMUs to the passive optical marker shields to directly compare the optical tracking system with our sensor-based system.
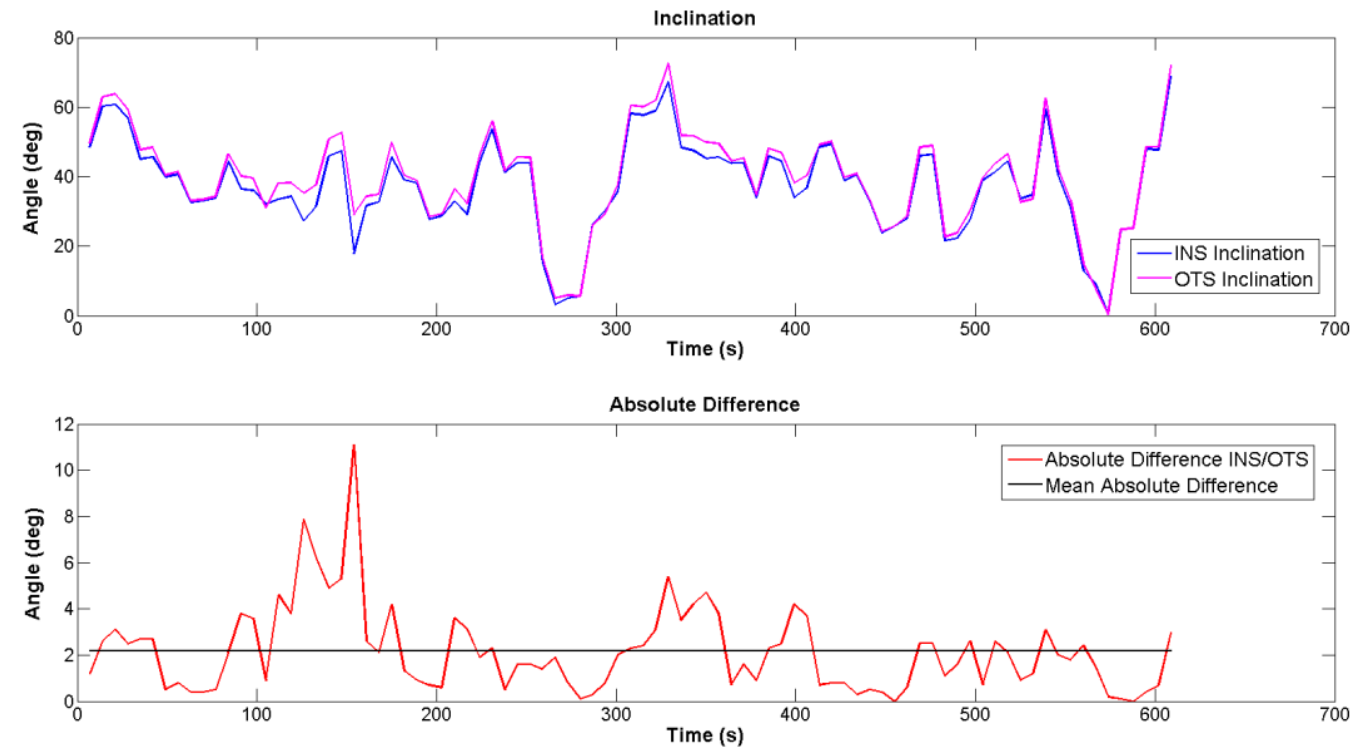

Figure 7: Top: Comparison of inclination values between our inertial sensor-based system (blue) and the current gold-standard, optical tracking (magenta) for one hip joint (2L). Bottom: The absolute difference between the inertial-based and the optical tracking-based system (red). The mean absolute difference is shown in black.
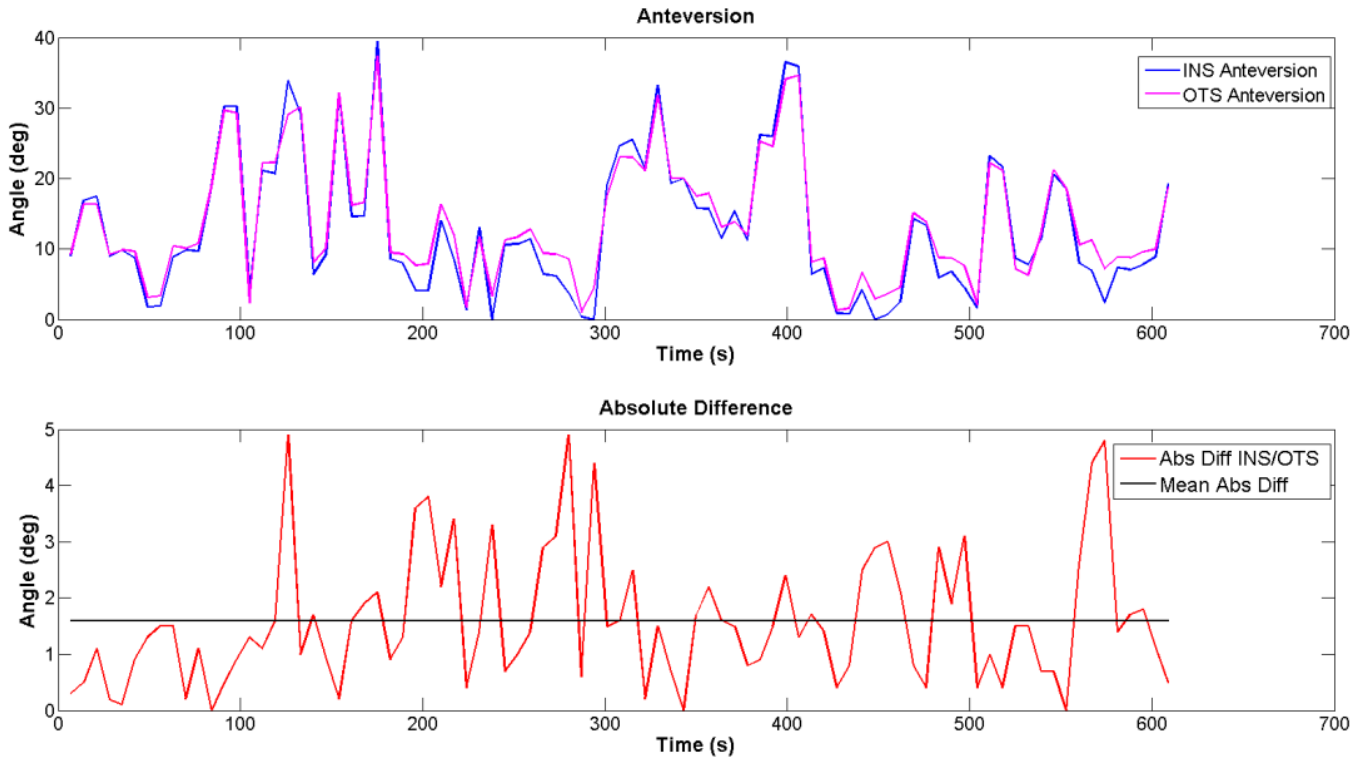

Figure 8: Top: Anteversion comparison between our system (blue) and the optical tracking-based system (magenta) for one hip joint (2L). Bottom: The absolute difference between the inertialbased and the optical tracking- based system (red). The mean absolute difference is shown in black. 


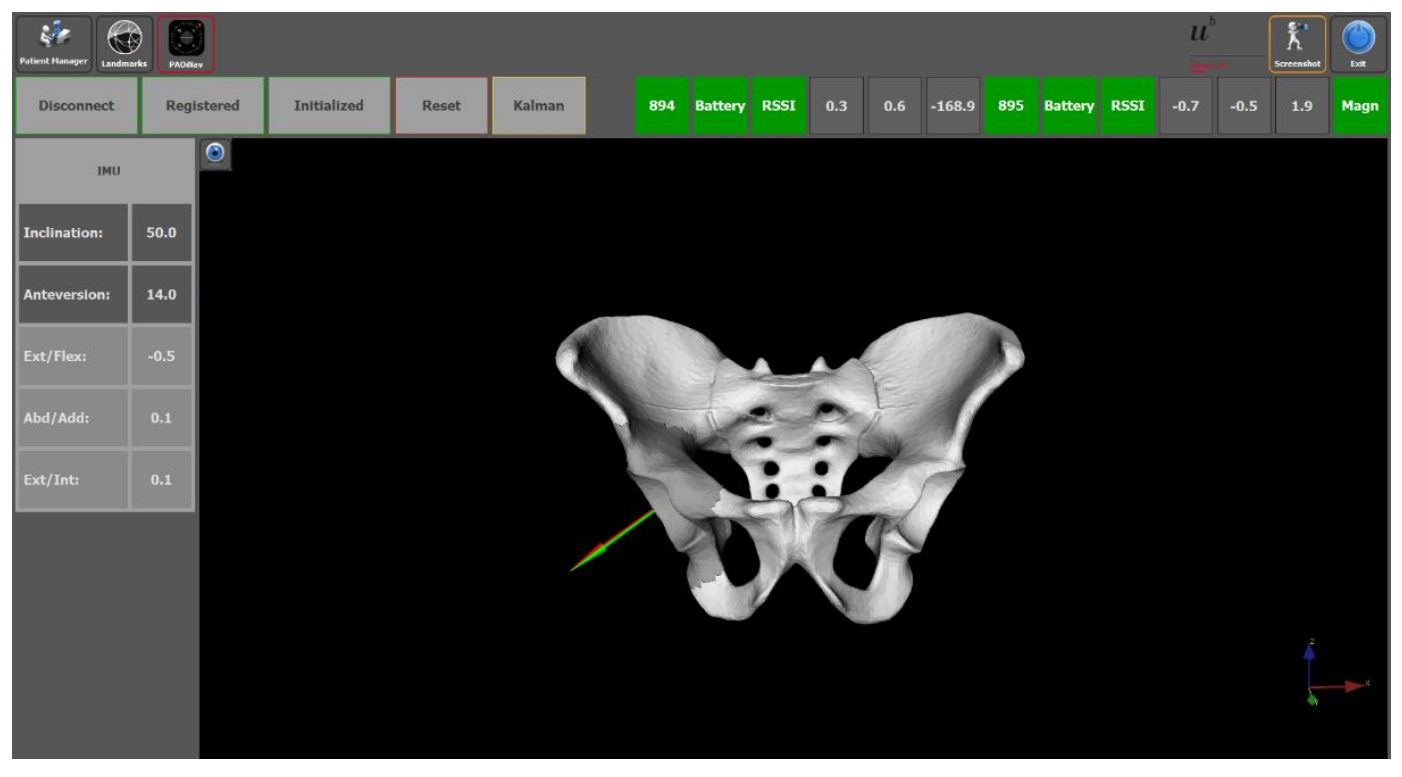

Figure 9: Our application to visualize the re-orientation of the acetabular fragment. The different steps like connection, APP registration and initialization of the starting orientation can be started using buttons at the top. Battery level and signal strength of the sensors is indicated using green (or red) marks at the top. The computed orientation values (here Euler angles) are displayed at the top right. If the magnetic field changes abruptly, it will be indicated at the top right. The updated inclination and anteversion values are shown on the side together with the decomposition into extension/flexion, abduction/adduction and external/internal rotation. The acetabular fragment is rotated on the screen to give the surgeon a $3 \mathrm{D}$ view of the current state including the starting acetabular cup plane normal (red vector) and the real-time updated current acetabular cup normal (green vector). 
Table 1: Experiment results for inclination. The letter next to the pelvis identification number represents the joint side ( $\mathrm{L}$ - left and $\mathrm{R}$ - right). The mean absolute, minimum and maximum differences is shown compared to the optical tracking system as well as correlation coefficient and p-value based on Wilcoxon rank sum test. There was no significant difference to the optical tracking system observed ( $\mathrm{p}>0.05)$ and correlation between the two system is very high $(\mathrm{c}>0.9)$.

\begin{tabular}{|c|c|c|c|c|c|}
\hline Pelvis & Mean Abs & Min Diff & Max Diff & Correlation & p-val \\
\hline $\mathbf{1 R}$ & 0.9 & 0.0 & 3.8 & 0.996 & 0.877 \\
\hline $\mathbf{1 L}$ & 1.0 & 0.1 & 3.6 & 0.998 & 0.782 \\
\hline $\mathbf{2 R}$ & 1.9 & 0.0 & 7.6 & 0.987 & 0.735 \\
\hline $\mathbf{2 L}$ & 2.2 & 0.0 & 11.1 & 0.991 & 0.239 \\
\hline $\mathbf{3 R}$ & 1.5 & 0.1 & 7.4 & 0.996 & 0.984 \\
\hline $\mathbf{3 L}$ & 2.3 & 0.0 & 5.9 & 0.983 & 0.612 \\
\hline $\mathbf{4 R}$ & 1.7 & 0.1 & 5.7 & 0.996 & 0.711 \\
\hline $\mathbf{4 L}$ & 2.3 & 0.0 & 5.2 & 0.987 & 0.697 \\
\hline
\end{tabular}


Table 2: Experiment results for anteversion. The letter next to the pelvis identification number represents the joint side ( $\mathrm{L}$ - left and $\mathrm{R}$ - right). The mean absolute, minimum and absolute difference is shown compared to the optical tracking system as well as the correlation coefficient and p-value based on Wilcoxon rank sum test. There was no significant difference to the optical tracking system observed $(\mathrm{p}>0.05)$ and correlation was very high $(\mathrm{c}>0.9)$.

\begin{tabular}{|c|c|c|c|c|c|}
\hline Pelvis & Mean Abs & Min Diff & Max Diff & Correlation & p-val \\
\hline $\mathbf{1 R}$ & 0.6 & 0.0 & 2.0 & 0.995 & 0.991 \\
\hline $\mathbf{1 L}$ & 1.4 & 0.0 & 3.7 & 0.994 & 0.520 \\
\hline $\mathbf{2 R}$ & 0.6 & 0.0 & 1.3 & 0.999 & 0.837 \\
\hline $\mathbf{2 L}$ & 1.6 & 0.0 & 4.9 & 0.988 & 0.255 \\
\hline $\mathbf{3 R}$ & 0.9 & 0.0 & 4.0 & 0.993 & 0.689 \\
\hline $\mathbf{3 L}$ & 2.9 & 0.0 & 7.6 & 0.945 & 0.253 \\
\hline $\mathbf{4 R}$ & 2.1 & 0.1 & 5.6 & 0.965 & 0.729 \\
\hline $\mathbf{4 L}$ & 1.9 & 0.0 & 5.3 & 0.963 & 0.156 \\
\hline
\end{tabular}


Table 3: Comparison of the rotation matrices (used to rotate the fragment) acquired every 20s during reorientation. The letter next to the pelvis identification number represents the joint side $(\mathrm{L}$ - left and $\mathrm{R}$ - right). Column 1: the comparison is performed using quaternion representation. Equation 20 should be satisfied if the two quaternions from the two systems represent the same orientation. Column 2 and 3: if our assumption of a stable magnetic field environment is valid, then there should be almost no change observable of the magnetic field vector in Earth-coordinate system. The standard deviation is shown for each sensors (attached to pelvis and attached to fragment during reorientation).

\begin{tabular}{|l|c|c|c|}
\hline Pelvis & $\begin{array}{c}\text { Quaternion } \\
\text { Comparison }\end{array}$ & $\begin{array}{c}\text { Magnetic Field } \\
(\text { std }- \text { Pelvis })\end{array}$ & $\begin{array}{c}\text { Magnetic Field (std - } \\
\text { Fragment) }\end{array}$ \\
\hline $\mathbf{1 R}$ & $0.9996 \pm 0.0004$ & {$[0.003,0.003,0.003]$} & {$[0.016,0.016,0.018]$} \\
\hline $\mathbf{1 L}$ & $0.9994 \pm 0.0007$ & {$[0.003,0.006,0.003]$} & {$[0.007,0.015,0.009]$} \\
\hline $\mathbf{2 R}$ & $0.9995 \pm 0.0003$ & {$[0.004,0.006,0.003]$} & {$[0.005,0.008,0.009]$} \\
\hline $\mathbf{2 L}$ & $0.9987 \pm 0.0037$ & {$[0.004,0.005,0.003]$} & {$[0.007,0.007,0.010]$} \\
\hline $\mathbf{3 R}$ & $0.9995 \pm 0.0010$ & {$[0.003,0.005,0.003]$} & {$[0.007,0.023,0.014]$} \\
\hline $\mathbf{3 L}$ & $0.9986 \pm 0.0010$ & {$[0.006,0.007,0.003]$} & {$[0.006,0.033,0.009]$} \\
\hline $\mathbf{4 R}$ & $0.9993 \pm 0.0006$ & {$[0.003,0.014,0.003]$} & {$[0.006,0.016,0.017]$} \\
\hline $\mathbf{4 L}$ & $0.9992 \pm 0.0006$ & {$[0.003,0.003,0.003]$} & {$[0.005,0.009,0.011]$} \\
\hline
\end{tabular}

